\title{
MANUFACTURING ARCHITECTURE DESIGN USING DISCRETE MATERIAL FLOW MANAGEMENT
}

\author{
Cotet, C. E.; Popa, C. L. \& Anghel, F. \\ University POLITEHNICA of Bucharest, IMST Faculty, MSP Department, \\ UPB-PREMINV Research Centre, \\ 313, Splaiul Independentei, Sector 6, Cod 060042, Bucharest, Romania \\ E-Mail: costel@mix.mmi.pub.ro, laur.popa79@gmail.com, florina@mix.mmi.pub.ro
}

\begin{abstract}
The main problem treated in this paper is that if we want to optimize a network of manufacturing systems design using discrete material flow management we need a new algorithm different from the one used for a single manufacturing system. For a single manufacturing system we usually use discrete material flow simulation to identify and eliminate bottlenecks where the flow is slowed down or blocked in order to increase the productivity. For a network of manufacturing systems material flow concentrators could be the bottlenecks found in one of the manufacturing systems using this classical discrete material flow simulation but it also could be a new different one. We focus in this paper on the algorithm we propose to solve this problem of identifying and eliminating not the bottlenecks of each manufacturing system but of the entire network. A case study of multipolar synchronous simulation (as we named our proposed algorithm) is presented in order to illustrate across a tree nodes manufacturing network how this new algorithm works. (Received in April 2009, accepted in June 2009. This paper was with the authors 1 month for 1 revision.)
\end{abstract}

Key Words: Simulation, Virtual Enterprise, Discrete Material Flow, Manufacturing Systems, Productivity.

\section{INTRODUCTION}

We define diffused manufacturing systems as architectures with more than two work points connected by transport \& transfer systems and using deposits at local or system level. We define then concentrate manufacturing systems as architectures based on a single work point surrounded \& assisted by transport, transfer \& deposit facilities [1]. Diffused as well as concentrated manufacturing systems could be mass production, batch production and job shops [2]. We agree here with the thesis that within the class of stochastic simulation models, one further distinction is necessary: simulations can be either terminating (sometimes called finite) or nonterminating in nature, with specific algorithms for each category [3].

We consider a material flow and process synchronous simulation the simulation of a model where at the level at the work point the process simulation is concomitant with the material flow simulation [4]. We define as multipolar synchronous simulation the integrated monitoring system of more than two material flow simulations interconnected in an enterprise network architecture [5]. We agree that virtual enterprises (VE) could be defined as a network of enterprises collaborating to produce a single product during a project cycle time [6]. In VE manufacturing design optimization, the simulation of the system material flow must succeed to CAD (computer aided design), CAE (computer aided engineering) and CAM (computer aided manufacturing) analyses [7]. For a designed manufacturing architecture it is always useful to simulate the material flow conduct before applying our design into practice in order to avoid potential flow concentrators (bottlenecks) generating low productivity or even 
blockage. Flow concentrators also generate under utilization of the structural elements of the manufacturing system leading to financial loss [8].

\section{MANUFACTURING ARCHITECTURE DESIGN REMODELLING}

In discrete material flow simulation we can create a model (that will contain: machines, parts, tools, etc.) for the whole manufacturing system based on data transmitted by the system modelling department (Fig. 1). The purpose is to achieve an optimum configuration for the system, regarding machines placement in the working place, the parts manufacturing order, etc. Having all the structural manufacturing elements (work points, transfer and transport systems, buffers, etc.) modelled, we can easily see all the operations and production phases, if it's necessary for the operator to intervene, the appearance of eventual problems, etc.

After the material flow simulation analyzing the reports of all the structural manufacturing elements activity we can identify eventual bottlenecks where the material flow is slowed or blocked.

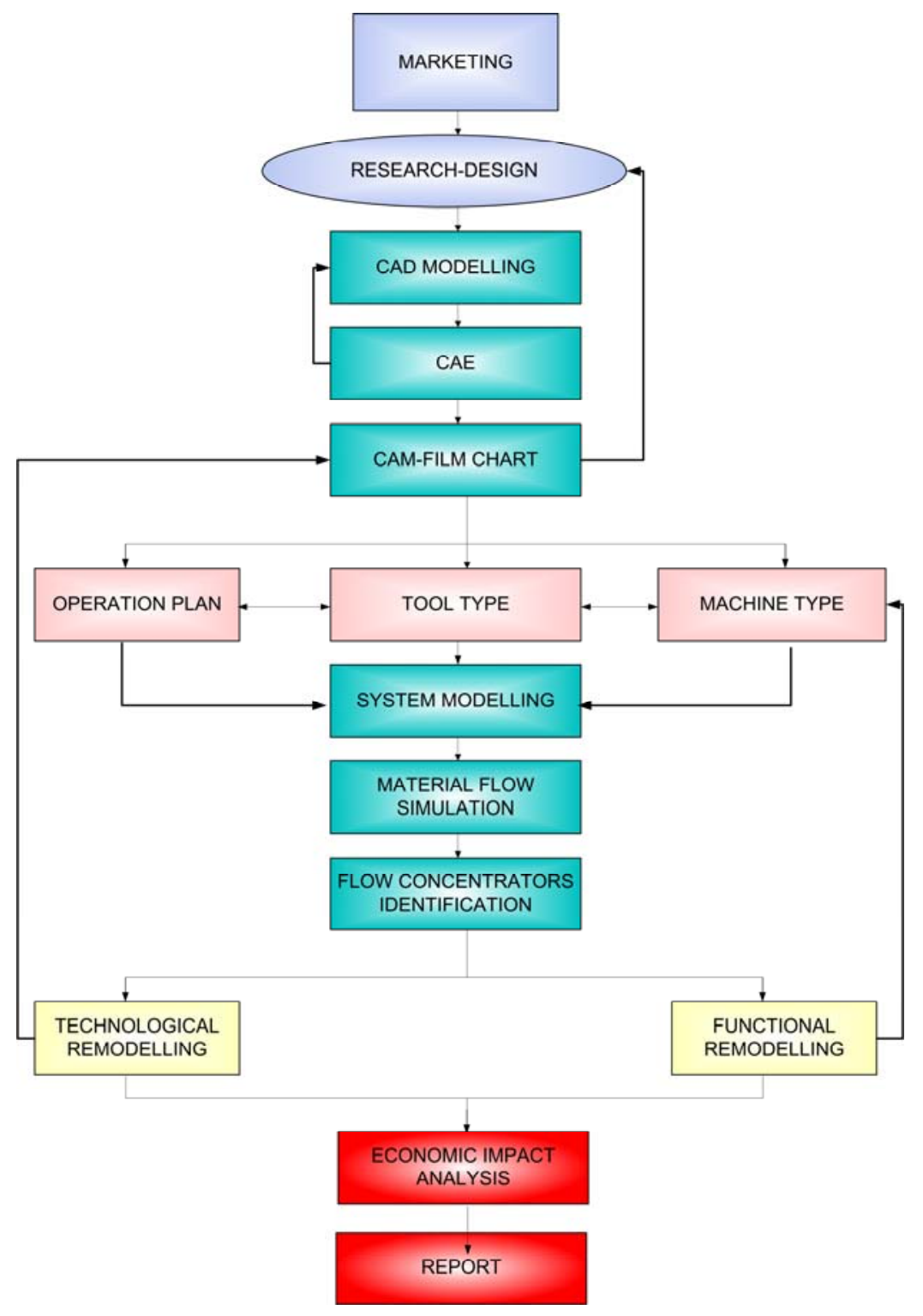

Figure 1: Methodology of modelling and simulation for the design and fabrication of a product. 
In order to eliminate those concentrators and increase the productivity we need to choose between:

- A functional remodelling; it consists in changing some of the machines placement, the order of some operations, the speed of some conveyors or manufacturing times;

- A technological remodelling; it consists in reconsidering all the system data: the type of the machines, tools, materials used etc. The new system is remodelled and a material flow simulation for the system is done.

If we had proposed a manufacturing architecture modification as a solution for eliminating flow concentrators we need to perform a second simulation in order to validate the optimized manufacturing architecture design by obtaining an increased productivity. Last but not least a financial analysis must confirm the profitability of the manufacturing optimized architecture. That means that the productivity gain covers the expenses implied by the functional or technological remodelling.

Three levels of integration are defined in the multipolar synchronous simulation algorithm:

At the first level main parameters for the work points modelling in material flow simulation are provided form CAM simulations describing the manufacturing process. This way the material flow simulator is integrating the process simulation results at the level of the work point in order to provide a complete model of the manufacturing system.

At the second level the terminating simulation algorithm (mainly used in VE related architectures) is applied for each manufacturing system of the virtual enterprise network.

At the third level the virtual enterprise material flow is simulated. In this model the work points are the terminating simulation models describing the virtual enterprise partners manufacturing systems material flows. At this level the complexity of synchronizing process and material flow simulation is higher because the stochastic laws describing the MTBF (mean down interval) and MTTR (mean repair time) [9] for every work point for every industrial partner activating as a virtual enterprise network node must be corroborate with the laws describing the integrated multipolar model work points (describing the material flow at the level of each enterprise as well as for the entire virtual enterprise architecture).

\section{A PRELIMINARY SADT MODEL}

The case study that allowed us to test our model was provided by one of our research projects implying three industrial partners working in a virtual enterprise environment. Two of them are products suppliers and the third one represents the enterprise where the parts are assembled, thus resulting the final product.

Each industrial partner can run a local material flow simulation in order to verify if his manufacturing system can realise its functions as a node of the VE network. Then, all simulations can be integrated in a multipolar simulation model that will be used to optimise the entire system productivity rates.

As one can see in Fig. 2 the VE architecture network used as a case study integrates as nodes three flexible manufacturing systems (FMS), as follows: FMS1 and FMS2 will be the components suppliers and FMS3 will be the system that assembles these components. FMS 1 and FMS2 are diffused manufacturing system and FMS3 is a concentrated manufacturing system. We considered the three partners as a network and we did not used a model based on supply chain simulation for the suppliers of FMS1 and FMS2 [10].

In order to describe the VE system network relationships and to find solutions to possible conflicts in the system we used SADT (Structured Analysis and Design Technique). In Fig. 2 partners $\mathrm{A}$ and $\mathrm{B}$ correspond to FMS1 and FMS2, and the assembly point is represented through $\mathrm{C}$ corresponding to FMS3. 


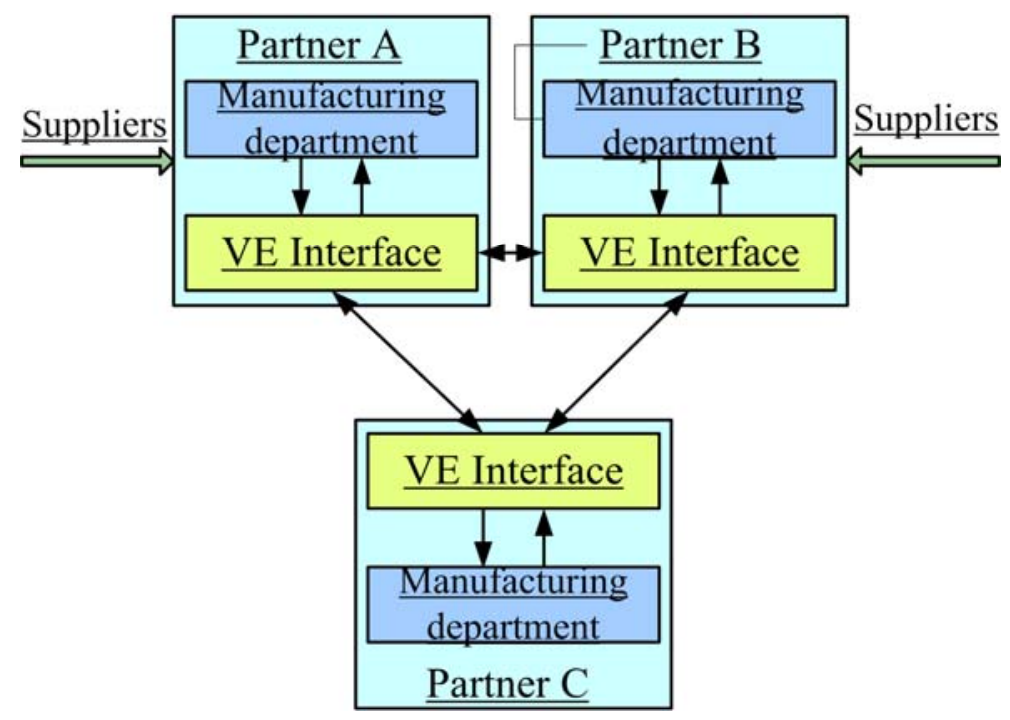

Figure 2: Virtual enterprise architecture for a study case.

Before running a material flow simulation we must define the structure of the material flow (tools and parts involved in the process for our case study) in each FMS as a node of the network and at the level of the entire VE. As well the structure of each FMS (work points, buffers, human resources, transport and transfer elements) must be defined.

The entries in the system are represented by the parameters of machine tools, tools and parts and the exit is the optimised architecture of the system. The main limitations of the system are (Fig. 3, element A, B, C): execution plans, available execution phases and the CAD - CAM - CAE system ability to analyse the working behaviour of machine-tools, parts and tools (Fig. 3, elements D, E and F). The simulation will be done using specialised software (Fig. 3, element G), which includes two modules as a mechanism for transforming the inputs in outputs. In Fig. 3 we can see the first datagram of the SADT algorithm. In order to apply this algorithm we need to define the studied system according with the following steps:

- Identify the elements in the system;

- Identify the inputs and outputs of the system;

- Evaluate limitations and mechanisms involved in the system functioning.

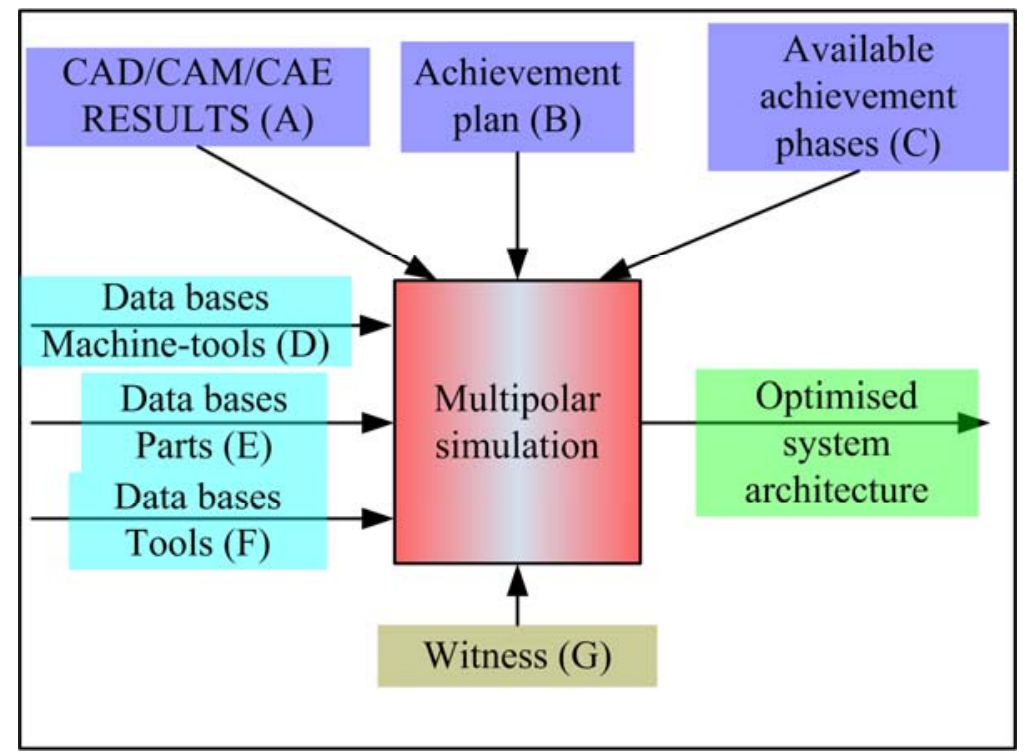

Figure 3: First phase in SADT algorithm. 


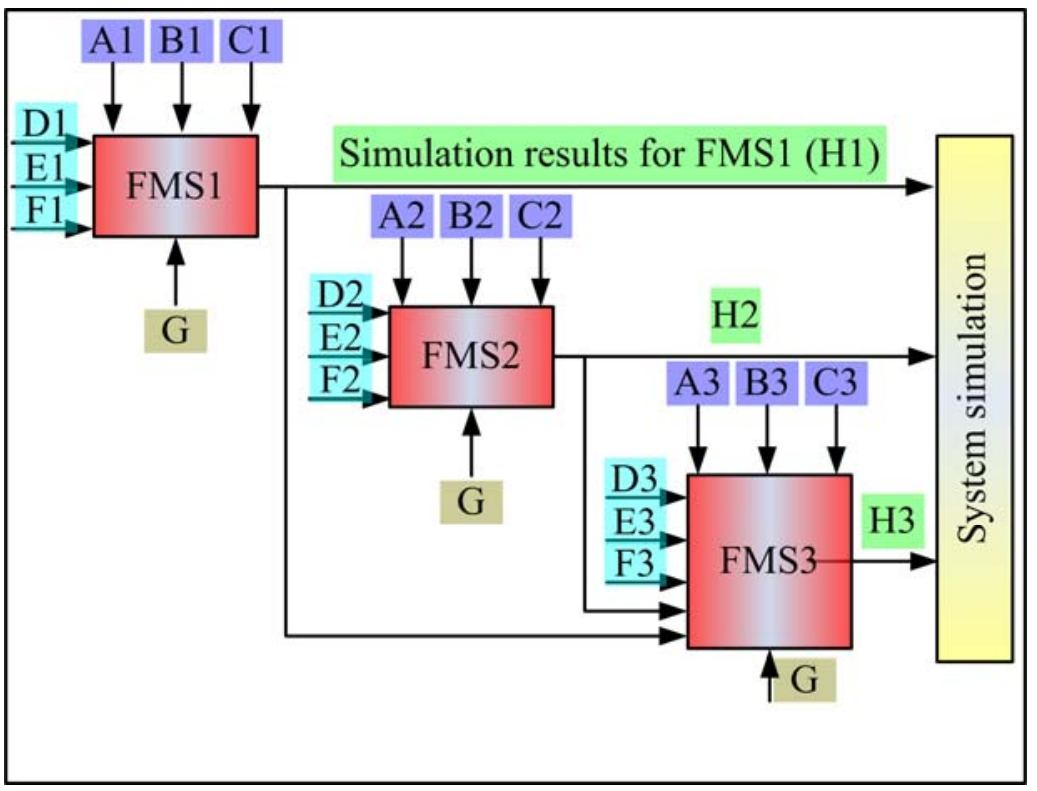

Figure 4: Second phase in SADT algorithm.

The next phase in the SADT algorithm (Fig. 4) is to identify the role of each system's element in the VE architecture:

- Realise a hierarchy between elements;

- Define the structure for each element;

- Define the relations between elements.

It was necessary to establish a hierarchy between elements in order to identify the element which has a determinant role in the system balance and is surrounded by all limitations and interactions present in system. According to the datagram presented in Fig. 4 this system was defined as FMS3, the virtual enterprise node in which the assembly takes place.

Analyzing all the main entries, (machine tools databases (D), parts databases (E) tools databases $(\mathrm{F})$ ) we determined that the main problem for a good parametric definition of the material flow is to define the part. The part definition can be influenced by the different limitations, representing the feedback given by the other elements from the system. The last definition is influenced by the definition of the available making process, the tools available and process validation. From the first stage to the final shape a part goes through many changes (regarding its geometry, tolerances, etc.) caused by limitations imposed by other system elements.

The part role will not be redefined but it's important to find a balance between the original project and the practical possibilities. Because of this, the final part concept will be done only after identifying the limitations induced by the validation process, which is done in the final phase.

Considering the data given above we define the structure for the three FMS models and the model for the entire system in order to run a simulation of the material flow. For achieving this several phases in SADT algorithm were necessary [11]. It is not our purpose to present here the entire datagram's succession. In those datagram all the working points were analysed (machine-tool, assembly work point), transfer and transport element (robots, conveyors), deposits, etc. Also we have studied the interactions with each system FMS.

We verified the SADT algorithm validity using "actigrams"; the model diagrams represented as verb cassettes sometimes with complementary data. The concordance of datagram and actigram representation results allowed us to perform the simulation based on the relationships established based on the SADT algorithm. 


\section{USING DISCRETE MATERIAL FLOW SIMULATION IN VIRTUAL ENTERPRISE MANUFACTURING NETWORKS DESIGN}

For improving the productivity we need to identify the material flow concentrators for each FMS and to the virtual enterprise as a system and we must propose a new architecture that will eliminate the concentrators. FMS1 and FMS2, which represent components suppliers, are diffused systems based on pallet operation. Pallet operation allows the improving of work cycles times; it reduces times and costs for installations. In these manufacturing architectures design we took into account that for diffuse systems the material flow can be extremely sensitive to the pallets number of the return bands, and also too many pallets can be more inefficient that less pallets. The operating time cycles and the auxiliary times are given in the equipment involved. FMS3 is a concentrated system.

Based on the Witness models we start to evaluate the FMS1 and FMS2 performances during simulation. For both FMS1 \& FMS2 the transfer from the conveyors to the machinetools is assured by robots associated with the machines and not represented in the model as separate entities. FMS1 have as structural elements: 4 machine-tools (M 1_1 to M1_4), 3 conveyors (C 1_1 to C1_3) and a buffer (B1). First we defined the parameters for each machine and conveyor and, after running the simulation we determined the place of the flow concentrators. Thus for FMS1 according to activity reports we can see blockages (at machine M1_2; the activity for this machine is blocked $92 \%$ of the time, $7 \%$ of the time is working, and $1 \%$ of the time is waiting for parts). Based on similar activity report in FMS1 we also can see blockages in C1_2, (its activity is blocked for $94 \%$ of the time, $5 \%$ of the time is working, and $1 \%$ of the time is waiting for parts). If we perform an activity report for M1_4 from FMS1 one will see that due to the blockages from the other machines M1_4 is working just $11 \%$ of the total time, $89 \%$ of the time is waiting for parts. Thus, the system appears to be inefficient. We must do a remodelling of the system, a new parameterisation of the system's components and eventually we can run a new simulation. Running the simulation of the FMS1 material flow after the material flow concentrator from M1_2 machine is eliminated; we can see by looking at the activity report that in this new configuration M1_2 machine is working $60 \%$ of the total time and $40 \%$ of the time is waiting for parts. If we also check the activity report for M1_4 machine an important improvement can also be noticed, (the machine is functioning $98 \%$ of the time, a big difference from the first case when it functioned only $11 \%$ of the time).

FMS2 have as structural elements: 5 machine-tools (M 2_1 to M2_5), 4 conveyors (C 2_1 to C2_4), a human operator (OP) and a buffer (B2). After the system material flow simulation different reports were generated in order to monitor the activity of each element from the system and for determining the elements where problems occur. If we study an activity report for M2_1 we can easily see that the machine is working $75 \%$ of the total time, $18 \%$ of the time it is blocked and $7 \%$ of the time the machine is not functioning, thus allowing the operator to intervene. The activity report for conveyor C2_1 show us that this working unit is blocked for $45 \%$ of the time, the conveyor is stopped because of the operator intervention for $5 \%$ of the time, thus the machine is functioning normally just $50 \%$ from total time. The activity report for M2_5 machine, the last one from this system is important because on this machine the last processing of the part takes place. This machine gives us important data regarding the total necessary time for processing a part in this system and the system productivity. From the chart we can see that the machine is waiting for parts $75 \%$ of the time and just $25 \%$ of the time the machine is processing parts. In the same way we generated an activity report for the human operator in the system. Thus, the operator is involved in repairing actions for $5 \%$ of the total time and in the rest of $95 \%$ of the time he is supervising the system activity and waits for intervention demands. 
So due to significant concentrators identified on M2_1 and C2_1 it resulted low productivity for this system (according to the report for M2_5 machine) and it is necessary to do a functional remodelling of the system and a new parameterise. The second system FMS2 was remodelled and a new simulation of the material flow was run. According to the reports generated we can notice an improvement of the system performances. The activity report for M2_1 machine after the system was remodelled show us that the blockages are reduced to half on this unit from $18 \%$ to $9 \%$, the machine functions normally for $70 \%$ of the time, but compared to the first simulation the time for operator interventions increases to $21 \%$.

Looking at the activity report for C2_1 conveyor after remodelling we can notice that the blockages presented in the previous simulation disappeared. Now the conveyor is working $55 \%$ of the time, $34 \%$ of the time its waiting for parts and the necessary time for human interventions is $11 \%$. From the report regarding the human operator activity we can see that his interventions doubled; the activity time percentage increases from $5 \%$ to $11 \%$. In the rest of $89 \%$ of the time the operator is supervising the system activity and waits for intervention demands in order to solve eventual problems occurred to machines from FMS2.

The productivity of FMS2 after remodelling can be seen from the activity report on M2_5 machine, which is the last machine where the parts are processed. The machine is processing parts $99 \%$ of the time and just $1 \%$ of the time is waiting for parts. Thus we can notice an improvement of the productivity after eliminating the concentrators discovered on the first simulation and a new parameterisation of the system elements.

In the third system FMS3 the two buffers B1 and B2 represent the inputs in the third system and the outputs of system 1 and 2 . The next elements present in the model are the two conveyors symbolised C3_1 and C3_2, and with M3_1 is symbolised the machine where the parts are assembled. The two parts are also represented and noted P and P2 and they are the result of the two previous systems FMS1 and FMS2. The human operator OP3 and a variable (nrpiece) counting the parts number in order to quantify the productivity rates are also elements of the model.

For identifying the material flow concentrators we generated a number of reports presented below:

- The activity report for C3_1 conveyor (the normal functioning time is $80 \%$ from the total time and $20 \%$ of the time it's blocked).

- The activity report for conveyor C3_2 (is blocked for $84 \%$ of the time and just $16 \%$ of the time is functioning normally).

- The activity report for M3_1 machine (89 \% of the time is functioning, $1 \%$ of the time is waiting for parts and $10 \%$ of the time reparations are in progress and other human activities).

After studying the reports and locating the flow concentrators, especially on C3_2 conveyor, we need to remodel the system taking into account that if the concentrators are not eliminated we must intervene on the system entries, so on the outputs of FMS 1 and 2. After remodelling studying the activity report for conveyor C3_2, we have seen that the blockage was reduced from $84 \%$ of the time to $19 \%$ of the time. Thus the normal functioning time is $80 \%$ of the total time, which indicates a major improvement of its activity. The activity report for M3_1 machine shows functioning $80 \%$ of the time and operator intervention $20 \%$ of the time. After analysing the remodelled FMS3 we also noticed an increasing of productivity for the whole virtual enterprise. By eliminating the concentrator from conveyor C3_2 we decreased the transfer and transport times for parts on M3_1 machine. The two components were delivered faster so the machine was reprogrammed to assembly faster. So the production cycle was significantly reduced. 
The presented case study, modelled and simulated in Witness validates the proposed algorithm for multipolar simulation, realising in fact a material flow simulation for a virtual enterprise manufacturing architecture.

One of the main problems that occur is calculating the necessary transportation time between the two supplier units and the virtual enterprise node (FMS3) where the assembly takes place. The working points are geographically de-located, so the distances are extremely important and it's more difficult to estimate a medium transportation time.

In the presented case study simulation medium times were used for FMS1 and FMS2 because it implicates relevant results and the variations are little, opposed to cases of parts transportation between systems (FMS3) where medium times can't be used because it varies from hours to weeks, depending on problems occurred on roads and distances. In such cases for a much more realistic aspect we need to calculate the transportation times using specific distribution laws.

\section{CONCLUSION}

In this paper we propose a new algorithm meant to optimize the manufacturing design not only for isolated manufacturing systems like in our previous researches but for manufacturing networks acting in VE architecture. We consider such an algorithm necessary because the flow concentrators for the network manufacturing are not always the bottlenecks identified for each of the manufacturing systems of the network acting isolated. In this new algorithm we perform a multipolar synchronous simulation where the elements of the model are simulating the behaviour of manufacturing systems and the transfer and transport systems are simulating the links between the FMS acting like nodes of the network. In order to describe the VE manufacturing system network relationships and to find solutions to possible conflicts in the system we used SADT methodology. To illustrate this algorithm we presented in this paper a case study performed in our research centre for a network of industrial partners. In this case study we evaluate a VE system architecture design based on three FMS acting like nodes in a manufacturing network. FMS1 and FMS2 are diffused manufacturing systems and FMS3 is a concentrated manufacturing system. First of all we performed the simulation of FMS1 and FMS2 manufacturing systems as isolated systems. After optimizing those manufacturing systems architecture based on eliminating the material flow concentrators we create the multipolar synchronous simulation model at the level of the FMS3 system. In this model the FMS1 and FMS2 products suppliers' models are acting like elements of the FMS3 model representing the enterprise where the parts are assembled, thus resulting the final product. The main purpose of the synchronous simulation was to use discrete material flow management to identify ways of improving the system configuration for productivity increase.

As in our previous material flow simulation based algorithms used for isolated FMS in this new algorithm one can analyze the results of the material flow simulation, identify the flow concentrator for the diffused manufacturing architecture and propose an architecture modification as a solution for this problem. A second simulation to validate the optimized architecture and the obtained increase of productivity is necessary as well as a financial analysis must confirm the profitability of such a solution.

The main innovative character of our new multipolar synchronous simulation algorithm is given by the three kinds of synchronic simulation identified and used by us in building our model.

First of all at the level of each manufacturing system the process simulation for each work point is synchronic with the material flow simulation describing the isolated system activity. Secondly the simulation of the material flow multipolar model and the simulation of material flow for each manufacturing system model are synchronous. Last but not least the integrated 
multipolar model is synchronizing the results of material flow and process simulation models for all the nodes of the virtual enterprise architecture the isolated FMS simulations acting like elements of the multipolar model (workpoint, buffers, etc.).

If we want to optimise virtual enterprise integrated behaviour it's not enough to run a discrete material flow simulation. The discrete material flow management is just one of the main integrated management systems for a virtual enterprise [12]. However the multipolar simulation, based on the model done using an SADT algorithm is very useful in order to optimize the manufacturing design of the VE architecture.

\section{REFERENCES}

[1] Cotet, C. E.; Dragoi, G. (2003). Material Flow Management in Validating Concentrate and Diffused FMS Architectures, International Journal of Simulation Modelling, Vol. 2, No. 4, 109120

[2] Dhouib, K.; Gharbi, A.; Ayed, S. (2009). Simulation based throughput assessment of nonhomogeneous transfer lines, International Journal of Simulation Modelling, Vol. 8, No. 1, 5-15, doi:10.2507/IJSIMM08(1)1.111

[3] Sanchez, S. M. (2001). ABC's of output analysis, Proceedings of the 2001 Winter Simulation Conference, 30-38

[4] Cotet, C. E.; Dragoi, G.; Abaza, B. F. (2008). Groundhog day versus butterfly effect revisited in discrete material flow management, Annals of DAAAM for 2007 \& Proceedings of the 19th International DAAAM Symposium "Intelligent Manufacturing \& Automation: Focus on Next Generation of Intelligent Systems and Solutions", 22-25 ${ }^{\text {th }}$ October 2008, Trnava, Slovakia, 315316

[5] Cotet, C. E.; Dragoi, G.; Abaza, B. F. (2007). Multipolar synchronous material flow \& process simulation in difussed manufacturing systems, Annals of DAAAM for 2007 \& Proceedings of the 18th International DAAAM Symposium "Intelligent Manufacturing \& Automation: Focus on Creativity, Responsibility and Ethics of Engineers", Zadar, Croatia, 179-180

[6] Tolle, M.; Bernus, P.; Vesterager, J. (2002). Reference models for virtual enterprises, Collaborative Business Ecosystems and Virtual Enterprises, Camarinha - Matos, L. M. (Editor), Kluwer Academic Publishers, Boston, 3-10

[7] Lee, K. (1999). Principles of CAD/CAM/CAE Systems, Addison Wesley Longman, Reading

[8] Gill, A. (2008). Identifying potential bottlenecks through activity under-utilization cost, International Journal of Simulation Modelling, Vol. 7, No. 4, 165-175, doi:10.2507/IJSIMM $\underline{07(4) 1.104}$

[9] Ilar, T.; Powell, J.; Kaplan, A. (2008). Simulation of production lines - The importance of breakdown statistics and the effect of machine position, International Journal of Simulation Modelling, Vol. 7, No. 4, 176-185, doi:10.2507/IJSIMM07(4)2.105

[10] Wang, Q.; Ingham, N. (2008). A discrete event modelling approach for supply chain simulation, International Journal of Simulation Modelling, Vol. 7, No. 3, 124-134, doi:10.2507/IJSIMM07 (3) 2.100

[11] Rigal, J. F.; Ispas, C.; Cotet, C. E. (1998). Using SADT methodology for drilling process integrated in Pro/Engineer, First International Symposium on Concurrent Enterprising, 4-6 June 1998, Sinaia, 41-45

[12] Botezatu, C. P.; Carutasu, G.; Botezatu, C. (2008). Building Integrated Management Systems, DAAAM International Scientific Book 2008, Katalinic, B. (Editor), DAAAM International Vienna, Austria, 125-136, doi:10.2507/daaam.scibook.2008.11 